\title{
PALEOBIOGEOGRAFÍA DEL ARRIBO DE MAMÍFEROS SURAMERICANOS AL SUR DE AMÉRICA CENTRAL DE PREVIO AL GRAN INTERCAMBIO BIÓTICO AMERICANO: UN VISTAZO AL GABI EN AMÉRICA CENTRAL
}

\author{
PALEOBIOGEOGRAPHY OF THE ARRIVAL OF SOUTH AMERICAN MAMMALS \\ TO SOUTHERN CENTRAL AMERICA PRIOR TO THE GREAT AMERICAN \\ BIOTIC INTERCHANGE: \\ A LOOK AT THE GABI IN CENTRAL AMERICA
}

\author{
César A. Laurito ${ }^{1 \& 2 *} \&$ Ana L. Valerio ${ }^{2}$ \\ ${ }^{1}$ INA, Instituto Nacional de Aprendizaje \\ ${ }^{2}$ Departamento de Historia Natural, Museo Nacional de Costa Rica, \\ apdo. 749-1000, San José \\ *Autor para contacto: cesarlaurito@ice.co.cr
}

(Recibido: 15/03/2012 ; aceptado: 11/06/2012)

\begin{abstract}
In reference to the discovery of new fossil xenarthrans that was made in the Curré Formation, a paleobiogeographical analysis of the early Hemphillian age vertebrates will be made for the fossiliferous location of San Gerardo de Limoncito in southern Costa Rica. This vertebrate locality has a mixture is mainly formed by vertebrates from the Palearctic, Nearctic and Neotropical regions and it records the early arrival of South American mammals to the Southern Central America region, not known until now.

The arrival of South American mammals to the Panama Region was due to a series of factors such as the development of the Costa Rica-Panamá Island Arc by subduction processes, the collision and the deformation of the Island Arc with the North-Western Colombia territories and the prevailing climate factors which stimulated the predominance of warm, humid and basal forests, both in Colombia and in southern Central America. This permitted the xenarthrans, which are considered strong swimmers and islands hoppers to migrate to the North.

These conditions on the other hand, limited the migration to the South of the North American mammals, whose arrival in South America was postponed until the Blancan age with drier climatic conditions and the new land corridor that was established in the region.

Additionally, the current state of the Great American Biotic Interchange in Central America (GABI) is analyzed
\end{abstract}

Key words: Early Hemphillian, herald mammals, xenarthrans, Pliometanastes, Scirrotherium, Trionychidae, GABI, Costa Rica, Central America. 


\begin{abstract}
RESUMEN: A la luz de los nuevos registros fósiles de xenarthras en la Formación Curré, se realiza un análisis paleobiogeográfico de la fauna de vertebrados de la localidad fosilífera de San Gerardo de Limoncito en el sur de Costa Rica de edad Hemphilliano temprano. Esta asociación faunística se caracteriza por la mezcla de vertebrados de origen Paleártico, Neártico y Neotropical, a la vez que registra el arribo más temprano, hasta ahora conocido, de mamíferos suramericanos al sur de América Central. La llegada de mamíferos suramericanos al Istmo de Panamá se debió al acortamiento de los pasos oceánicos entre las islas, producto de la subducción normal asociada al Arco de Islas, el rápido levantamiento que experimentó el sur de América Central debido a la colisión del sur del Arco de Islas Costa Rica-Panamá con el noroeste de Colombia y por los elementos climáticos prevalecientes en esa época, que favorecieron el predominio de bosques húmedos, cálidos y basales tanto en el noroeste de Colombia como en el sur de América Central, lo que permitió a los xenarthras, considerados fuertes nadadores y saltadores de islas migrar al norte. Estas condiciones climáticas por el contrario, limitaron la migración al sur de los mamíferos norteamericanos, cuyo arribo a América del Sur se postergó hasta el Blancano con la aparición de condiciones más secas y el establecimiento de un corredor terrestre continuo. Adicionalmente se analiza el estado actual del Gran Intercambio Biótico entre las Américas en América Central.
\end{abstract}

Palabras clave: Hemphilliano temprano, mamíferos heraldos, Pliometanastes, Scirrotherium, Trionychidae, GABI, Costa Rica, América Central.

\section{ANTECEDENTES}

Siempre se tuvo la idea de que para comprender la importancia de los xenarthras en el intercambio faunístico entre las Américas y su patrón de distribución, era necesario realizar trabajo de campo en América Central a fin de buscar evidencia que aclare su ecología (McDonald, 2005).

Junto con los restos de gomphotherios, tayassuidos, camellos, caballos y reptiles encontrados en la localidad de San Gerardo de Limoncito, se recuperaron restos de Pampatheriidae, Megalonychidae, Megatheriidae y Mylodontidae; éste conjunto de xenarthras representan el "stock" más temprano de inmigrantes sudamericanos hallado en América Central y esta localidad es hasta el momento, la localidad fosilífera continental con la evidencia más temprana Pre - Gran Intercambio Biótico entre las Américas vía el Istmo de Panamá.

Hasta ahora todas las faunas del Mioceno de Mesoamérica mostraban una estricta afinidad norteamericana (Ferrusquía-Villafranca, 2003), la fauna de San Gerardo de Limoncito (Apéndice) viene a cambiar de manera radical esta situación y para comprender la importancia que juega dicha fauna en el Intercambio Biótico entre las Américas, es necesario entender los escenarios paleogeográficos y las su- cesiones faunísticas de vertebrados del Mioceno de América Central, teniendo como contexto a algunas localidades fosilíferas de Sur y Norte América.

Recientemente Coates et al. (2004), han dilucidado parte del rompecabezas del desarrollo paleogeográfico del sur del Istmo de Panamá, fundamentados en el análisis de la historia sedimentaria, las sucesiones faunísticas de foraminíferos y otros invertebrados marinos, y los eventos tectónicos relacionados con el arribo y choque del Arco de Islas de América Central Meridional con la Placa Suramericana. Dichos autores reconocen 3 épocas claves en el desarrollo de la historia geológica de América Central Meridional (Fig. 1), identificadas como de Pre-colisión (Cretácico Superior, desde el inicio de la formación del Arco de Islas hasta el Mioceno Medio temprano, > 14,8 Ma), Colisión temprana o Syn-colisión (Mioceno Medio temprano hasta el Mioceno tardío, $<14,8$ Ma a 7,1 Ma) y Post-colisión (Mioceno tardío en adelante, $<7,1 \mathrm{Ma})$. A cada una de esas épocas de desarrollo paleogeográfico del Arco de Islas es posible asociarlas a un escenario paleobiogeográfico determinado, fundamentados en la faunas de tetrápodos terrestres e incluso es posible establecer una época correspondiente con el inicio del establecimiento del corredor biológico interamericano de previo al GABI $(<4,8-4,5 \mathrm{Ma})$ 


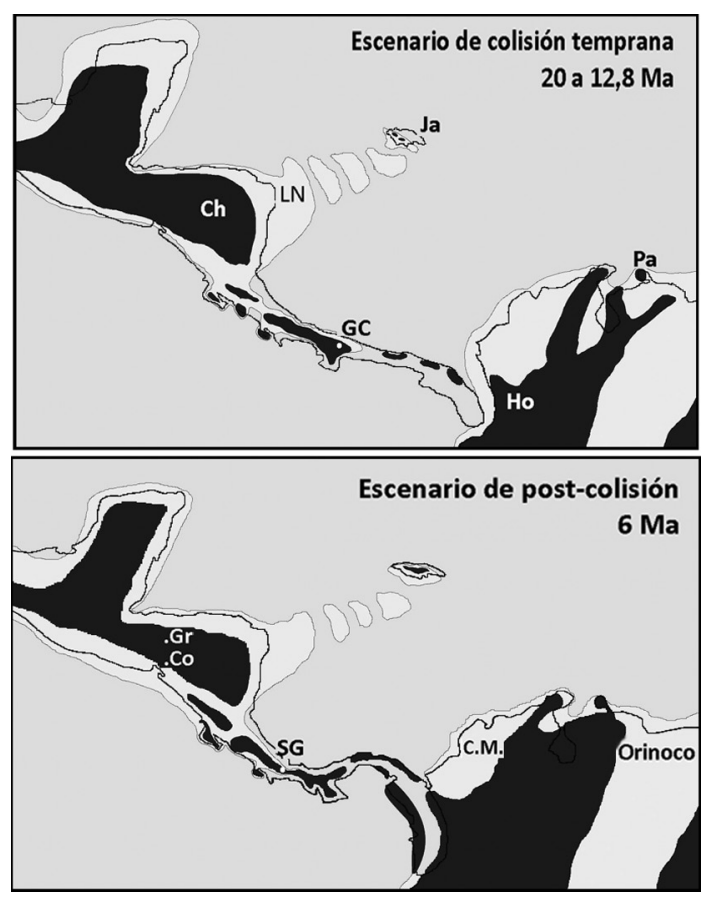

Fig. 1: Paleogeografía del área de estudio: Gr, Gracias; Co, Corinto; SG, San Gerardo; GC, Gaillard Cut ; Ho, Grupo Honda; Pa, Paraguaná, CM, Cuenca del Magdalena; Ch, Chortis y Ja, Jamaica.

de acuerdo con la sucesión de las faunas tempranas registradas en el Valle de México (CarranzaCastañeda \& Miller, 2004).

\section{Escenario faunístico de Pre-colisión $>$ 14,8 Ma}

No hay registro de vertebrados fósiles continentales en América Central Meridional para el Paleógeno, ni de previo al Oligoceno tardío. Sin embargo, cuando analizamos el Bloque Chortis, merece la pena señalar la presencia de dinosaurios ornitópodos en el Cretácico Medio de Honduras (Horne, 1994), que es muy anterior incluso al inicio de la formación del Arco de Islas Costa Rica - Panamá. Su ubicación en América Central se debió a la deriva del bloque Chortis que se desprendió de la Placa Norteamericana.

Otro registro fósil importante lo es el hallazgo del Rhinocerotoidea Hyrachyus sp. en el Eoceno temprano tardío o medio temprano de Jamaica
(Domning et al., 1997); lo que sugiere una conexión terrestre dentro del Bloque Chortis, entre Jamaica y América Central nuclear, que permitió la colonización de la isla por estos rinocerontes, conexión que empezó a desaparecer con la progresiva inundación del levantamiento de Nicaragua durante el Eoceno temprano o medio (Robinson, 1988).

Lucas (com. esc., 2012) comenta que "él duda que los rinocerontes fueran muy diversos y abundantes en esa época y que no alcanzaron América Central hasta el Mioceno Medio, de acuerdo al registro de Panamá". Sin embargo, nuestro criterio es que independientemente de que los rinocerontes fueran muy diversos o abundantes en el Eoceno, se debe tomar en cuenta que el Bloque Chortis conformó un terreno emergido continuo que conectó el Bloque Maya con Jamaica a través del Levantamiento de Nicaragua en el Eoceno temprano y medio, y ello lo confirman diversos autores, entre ellos Pindell (1994), Iturralde-Vinnent \& MacPhee (1999) e incluso en los modelos más viejos como el de Sykes et al. (1982), ya se visualizaba este tipo de conexión y ello explicaría eventualmente la dispersión vicariante del género Hyrachyus en Jamaica.

Es importante destacar que para este periodo y tan pronto como en el Oligoceno tardío y el Mioceno temprano, hace alrededor de 17,5-18,5 $\mathrm{Ma}$, los xenarthras suramericanos colonizaron las islas de Puerto Rico y Cuba, ello representa el ingreso más temprano de fauna suramericana en el Caribe (MacPhee \& Iturralde-Vinnent, 1994, 1995; MacPhee, 2005). Recientemente, se ha dado a conocer el hallazgo del gavial Aktiogavialis puertoricensis en el Oligoceno de Puerto Rico por Vélez-Juarbe et al. (2007) y no hay duda que esta especie derivó de los gaviales gryposuchinos suramericanos, su arribo a las Antillas Mayores sugiere algún grado de tolerancia al agua salada.

Por otro lado, solo se han identificado en el sur de América Central tres localidades fosilíferas portadoras de mamíferos terrestres, la fauna de Las Cascadas en la formación del mismo nombre y de edad Oligoceno tardío- Mioceno temprano y las localidades de Centenario y Gaillard Cut, pertenecientes a la Formación La Cucaracha de edad Mioceno Medio, todas afloran en la Zona del 
Canal de Panamá y son consideradas las faunas con registro de vertebrados terrestres más antiguas de América Central.

Dichas faunas se caracterizan por elementos de estricto abolengo norteamericano (sensu Reig, 1981), en general son formas del centro de México, las planicies costeras del Golfo de México, Florida, Dakota, Colorado, Maryland y California.

La fauna de la Formación Las Cascadas es notable por el registro de los Camelidae Aguascalientia panamaensis Rincón et al., 2012 y Aguascalientia minuta Rincón et al., 2012 de edad NALMA Arikareeano tardío de acuerdo con Rincón et al. (2012).

Entre los fósiles de la Formación La Cucaracha se hallan restos de Rodentia con la especie Texomys stewarti cf. Slaughter, 1981; Carnivora (Canidae y Amphicyonidae), Artiodactyla (Tayassuidae, Oreodontidae y Protoceratidae) y Perissodactyla (Equidae y Rhinocerotidae) cf. MacFadden (2006), MacFadden et al. (2010). También cuenta con un registro importante de tortugas fósiles, destacando los géneros Rhinoclemmys, Geochelone, Staurotypus y Trionychidae indeterminado (Cadena, 2009; Cadena et al., 2012).

La edad Nalma de la fauna de Centenario, de la cual se recuperaron únicamente dos especies de Tayassuidae "Cynorca" occidentale junto con otro Tayassuidae indeterminado, es Hemingfordiano temprano (He1) a Barstoviano temprano (Ba1) y la de Gaillard Cut es Arikareeano tardío (Ar4) Barstoviano temprano (Ba1), ninguna cuenta con elementos de origen sudamericano (Cuadro 1) (MacFadden, 2006; MacFadden et al., 2010).

La naturaleza y el gran porte de los mamíferos de esta fauna (a excepción del roedor) así como los géneros de tortuga representados, confirma que son formas estrictamente continentales y que su dispersión tan al sur como la Zona del Canal de Panamá solo fue posible mediante la existencia de un territorio peninsular (Kirby \& MacFadden, 2005), es decir, el Arco de Islas Costa Rica-Panamá conformaba una península unida al bloque Chortis y este se conectaba a su vez con la placa Norteamericana.

En este mismo periodo de Pre-colisión, se tiene la evidencia más temprana del arribo de un elemento norteño en América del Sur, el hallazgo se ubica en Venezuela en el estado de Lara, en la localidad fosilífera de Cerro La Cruz en la Formación Castillo de edad Mioceno Inferior (23,0 a 16,0 Ma). Se trata de la tortuga de caparazón blando Apalone sp. (Sánchez-Villagra et al., 2004) que es contemporáneo con el registro panameño de un Trionychidae indeterminado muy similar a Apalone (Cadena, 2009; Cadena et al., 2012).

La ruta de dispersión de los Trionychidae en general es bastante incierta y algunos autores han asumido una posible migración desde la península de La Florida al norte de Sudamérica vía las Antillas Mayores y Menores (Pritchard, 1984; Head et al., 2006). Pero, desde un inicio Wood \& Paterson (1973), sugirieron América Central como la ruta de dispersión más probable, ya que no hay evidencia de dispersión alguna vía las Antillas; ello se vino a confirmar con los registros fósiles de Trionychidae en el Mioceno Inferior de Panamá (Cadena, 2009; Cadena et al., 2012) y el Mioceno Superior (Hemphilliano Temprano, Hh1-Hh2) de Costa Rica (Laurito et al., 2005a).

La anterior evidencia, a excepción del Trionychidae, confirma que al menos entre los 20,0 y $14,0 \mathrm{Ma}$, el territorio emergido más austral del istmo de Panamá, constituía una península (Kirby \& MacFadden, 2005) que se encontraba bastante lejos del noroeste continental de América del Sur, separada por un canal interoceánico profundo denominado Estrecho de Bolívar (sensu Withmore \& Stewart, 1965), de allí que el hallazgo suramericano del Trionychidae Apalone, sugiere algún grado de tolerancia al agua salada por parte de las tortugas de caparazón blando.

\section{Escenario de Syn-colisión o colisión temprana $<14,8$ Ma a 7,1 Ma}

Durante esta etapa de colisión temprana cabe destacar el continuo registro de Trionychidae suramericanos procedentes de la formaciones Urumaco de edad Mioceno tardío (Wood \& Paterson, 1973) y Cubaguá del Mioceno tardío y Plioceno temprano de la isla Margarita (Head et al., 2006), ambas localidades de Venezuela. 
Cuadro 1

Cuadro sinóptico cronológico de ocurrencia de diversos taxa para América del Sur, América Central y América del Norte

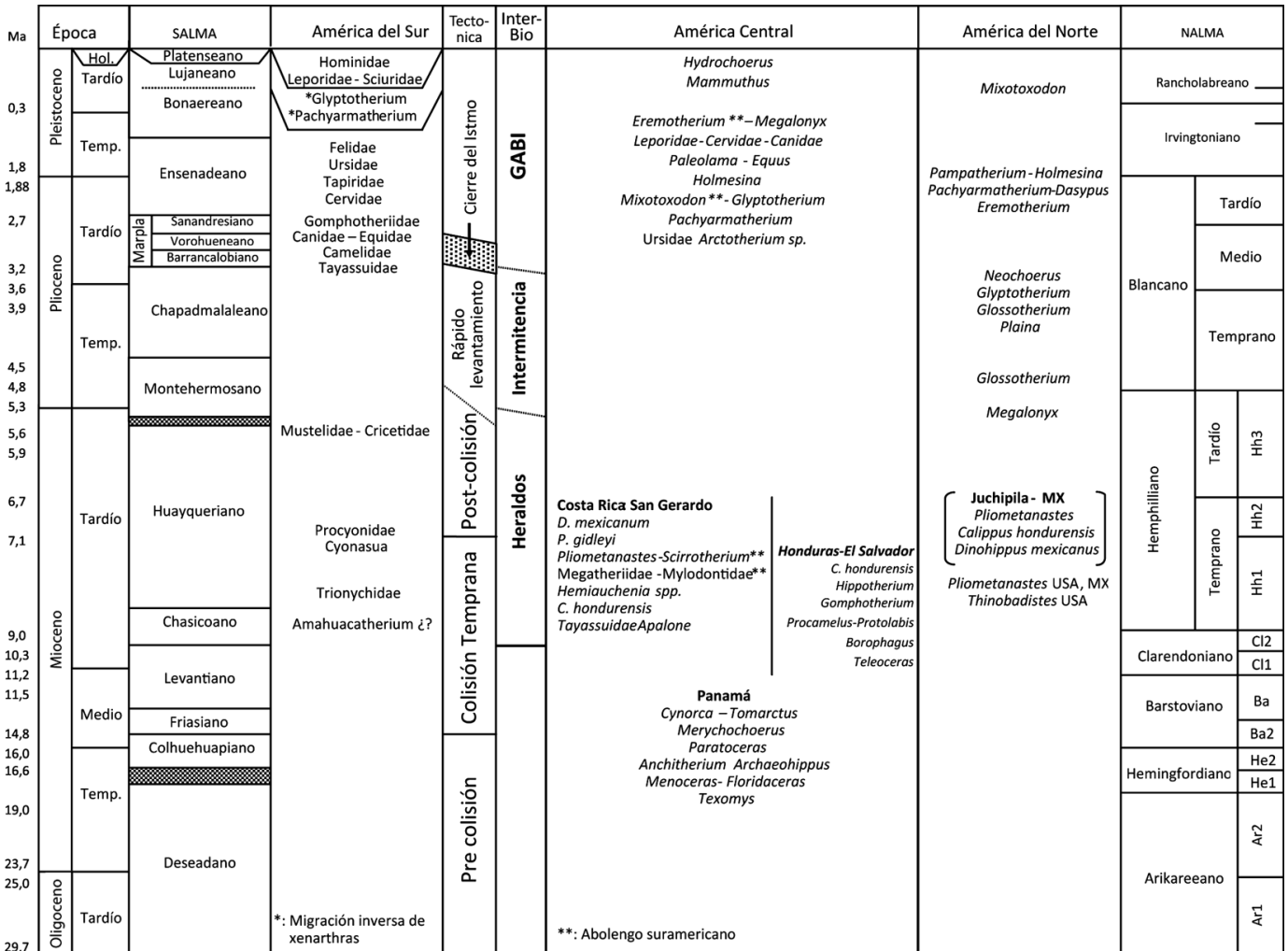

Aunque los Trionychidae actuales presentan intolerancia a los ambientes marinos y son eminentemente dulceacuícolas, por lo que se asume que tuvieron una capacidad de dispersión restringida en estos ambientes, la evidencia fósil indica que de una u otra manera los Trionychidae miocénicos fueron tolerantes a los ambientes marinos por el tipo de sedimento donde se han hallado, un ejemplo de ello son los Trionychidae del Mioceno tardío de la Formación Urumaco en Venezuela, todos hallados en depósitos costeros (Head et al., 1999, 2006; Sánchez-Villagra \& Scheyer, 2010), lo mismo sucede con el ejemplar panameño asociado a ambientes deltáicos (Cadena, 2009; Cadena et al., 2012) y el costarricense procedente de la Fauna de San Gerardo de Limoncito de la Formación Curré (Laurito et al., 2005a), ligeramente más tardío.
Fuera de los reptiles, el intercambio PreGABI está marcado por el arribo temprano tanto a América del Norte y del Sur de los primeros organismos Heraldos. Fue David Webb $(1976,1985)$, quién propuso el término "Heralds" o Heraldos en español, para identificar aquellos mamíferos precursores del Gran Intercambio Biótico entre las Américas, por comparación con aquellos hombres que portaban los estandartes de los ejércitos durante las batallas y que normalmente marchaban delante de estos, así él identifica a los mamíferos terrestres que han cumplido con la condición de Heraldos Norte y Suramericanos.

Entre los Heraldos del Norte se encuentra el hallazgo más temprano de un proboscídeo tretrabelodonto en América del Sur correspondiente a la especie Amahuacatherium peruvium RomeroPittman, 1996, un Gomphotheriidae del Mioceno tardío de la Amazonia peruana, su edad radiométrica 
de 9,0 Ma (Campbell et al., 2001), lo convierte en el mastodonte tetrabelodonte más antiguo de América del Sur, sin embargo existe reserva sobre la edad y taxonomía de este mastodonte, cuyos molares recuerdan más a los mastodontes dibelodontes más tardíos, como Stegomastodon (= Haplomastodon), que a los tetrabeledontes miocénicos de América del Norte (Cuadro 1). Por ejemplo, Ferretti (2008) argumenta que las únicas características de $A$. peruvium Romero-Pittman, 1996 que permitirían la distinción específica serían las defensas mandibulares y una muy baja mandíbula, pero el mismo autor, considera que solo se trata de una mala interpretación de material muy fragmentado y Alberdi et al. (2004) consideran que las defensas inferiores no están del todo presentes en la mandíbula. Su edad sugiere una aproximación paleogeográfica entre el sur de América Central y el norte de América del Sur. No hay duda que los mastodontes en general fueron buenos saltadores de islas y sus parientes actuales los elefantes se ha comprobado que son fuertes nadadores, con registros de hasta $48 \mathrm{~km}$ de la costa en el océano abierto (Johnson, 1980).

La edad del registro fósil de Amahuacatherium peruvium es casi 2,0 Ma más antiguo que el rango de ingreso a América del Sur de otros mamíferos inmigrantes norteamericanos como los Procyonidae del género Cyonasua, alrededor de los 7,0 a 7,5 Ma o edad Huayqueriano (sensu Patterson \& Pascual, 1972).

En relación con los inmigrantes suramericanos más tempranos a América del Norte, $A$. peruvium muestra contemporaneidad con los Xenarthra Pliometanastes (Megalonychidae) y Thinobadistes (Mylodontidae), ambos entre 8,09,0 Ma (Hemphilliano temprano -Hh1), hallados en la localidad fosilífera de McGehee en la península de La Florida sensu Morgan (2005). También, se han registrado en otras localidades de Estados Unidos, Pliometanastes se registra en el Hemphilliano temprano de California de acuerdo con Hirschfeld (1981), Hirschfeld \& Webb (1968), Webb (2006) y Thinobadistes en Texas (Webb, 1989) con edades similares. Recientemente, Carranza-Castañeda \& Miller (2004) registran el hallazgo de Pliometanastes en el estado de Nuevo León con una edad de alrededor de 8,0 Ma y el registro costarricense de Pliometanastes cf. protistus de edad Hemphilliano temprano (Cuadro 1)

Normalmente se aceptaba que la migración de estos Xenarthra a La Florida siguió una ruta de dispersión a través de las Antillas Menores y Mayores (Webb, 1985), ello parecía validarse por el notable registro de xenarthras fósiles en las Antillas. De hecho los registros más antiguos de xenarthras en las Antillas Mayores datan del Oligoceno temprano de Puerto Rico y Mioceno temprano de Cuba con alrededor de 17,5-18,5 Ma (cf. MacPhee \& Iturralde-Vinnent, 1994; White \& MacPhee, 2001; MacPhee et al., 2003), se acepta que arribaron durante el Oligoceno cuando el nivel del mar era extraordinariamente bajo y la configuración paleogeográfica de las Antillas Mayores permitió la conexión de estas con el levantamiento de Aves (Aves Ridge) y el norte de Sudamérica (MacPhee \& Iturralde-Vinnent, 1994, 1995; MacPhee, 2005). Pero no hay evidencia faunística de que las Antillas en general hayan estado alguna vez conectadas con las áreas continentales adyacentes en el Cenozoico mediante un territorio emergido continuo (Patterson \& Pascual, 1968) y es claro que la colonización de estas islas se dio por migración accidental (Simpson, 1956) de organismos saltadores de islas (Pascual, 2006). Además, este hecho confirma que los perezosos se pudieron dispersar a través de amplias barreras acuáticas y como la misma Hirschfeld (1985) concluyó, la dispersión por el Caribe de estos xenarthras los ubica como saltadores de islas, ya sea como fuertes nadadores o por medio de balsas naturales ( $=$ funerales vikingos).

Hirschfeld (1981) había concluido que existen diferencias taxonómicas entre las formas de las Antillas y las de América del Norte, de allí que la ruta de dispersión de las formas norteamericanas fue probablemente vía América Central, misma conclusión a la que llegaron CarranzaCastañeda \& Miller (2004). Ello fue demostrado por el registro de Pliometanastes cf. protistus en el Hemphilliano temprano de Costa Rica.

Por otra parte, cabe acotar en relación con los Procyonidae que no se han hallado fósiles en las Antillas Mayores y que los Procyonidae que han habitado las Antillas Menores, como la subespecie o especie Procyon gloveralleni, fueron 
introducidos por los humanos cuando estos arribaron a las islas (Helgen \& Wilson, 2003), lo que sugiere que probablemente la ruta de dispersión de los primeros procyonidos a América del Sur fue vía el Istmo de Panamá.

En América Central septentrional solo se registran 2 localidades fosilíferas correspondientes a la época de colisión temprana del arco de islas Costa Rica-Panamá. Las localidades de Corinto en El Salvador y el conjunto Año Nuevo, Rancho Lobo y Tepusuna o el Mangual de la Formación Gracias en Honduras que se caracterizan por una fauna continental estrictamente norteamericana, sin ningún elemento de origen suramericano, con edades del Mioceno Superior (sensu Webb \& Perrigo, 1984), más estrictamente Clarendoniano tardío a Hemphilliano temprano entre 9,0-7,0 Ma por la asociación de Hipparion plicatile (Leidy), Cormohipparion cf. occidentale y Calippus hondurensis (Olson \& McGrew, 1941) [Cl2 a Hh1], es decir, son ligeramente más viejas que la localidad fosilífera de San Gerardo de Limoncito en el sur de Costa Rica de edad Hemphilliano temprano (Hh 1 - Hh2) (Cuadro 1). A diferencia de las localidades de Honduras y El Salvador, la localidad costarricense cuenta con el registro tanto de elementos sudamericanos como norteamericanos.

\section{Escenario faunístico de Post-colisión $<7,1 \mathrm{Ma}$}

La fauna de San Gerardo de Limoncito de edad Hemphilliano temprano (8,5 a 6,5 Ma), se ubica en el final del escenario de Syn-colisión y al inicio del escenario de Post-colisión del sur del arco de islas Costa Rica-Panamá con el noroeste de Colombia y se enmarca justo en la época del Pre-Gran Intercambio Biótico entre las Américas o Pre-GABI (Cuadro 1). La sedimentología de esta localidad de la Formación Curré, sugiere un rápido levantamiento del fondo oceánico donde los sedimentos finos de la plataforma interna del Mioceno Medio, luego de una disconformidad erosiva, gradan rápidamente a sedimentos de abanico deltaico subacuático e infralitorales más gruesos del Mioceno Superior, conglomerados finos y areniscas medias respectivamente. Ello es coherente con el rápido levantamiento que experimentó el sur de América Central, producto de la colisión del sur del Arco de Islas Costa Rica -Panamá con el noroeste de Colombia (Kellogg \& Vega, 1995), evento que quedó registrado en múltiples formaciones sedimentarias del área (Duque-Caro, 1990a, b), como una discordancia erosiva en las cuencas de Chucunaque-Tuira y Sambu en la región del Darién sensu Coates et al. (2004). De acuerdo con dichos autores la discordancia se registró como un hiato erosivo que abarcó entre 8,6 y $7,1 \mathrm{Ma}$.

La localidad de San Gerardo, es la única localidad del país cuya fauna muestra una mezcla de mamíferos acuáticos y terrestres. Con respecto a los mamíferos acuáticos, destacan los cetáceos de agua dulce como el Iniidae Goniodelphis sp. y las formas marinas como Hadrodelphis sp., Orycterocetus sp. y Eurhinodelphis sp. que denotan afinidades epicontinentales paleocaribeñas con la península de Florida, Atlántico Norte y Mediterráneo (Valerio \& Laurito, en prensa), en tanto que los mamíferos terrestres a su vez constituyen una mezcla de ordenes de abolengo Norteamericano y Suramericano (Cuadro 1), siendo los xenarthras el único orden suramericano, representado por 4 familias: Megalonychidae, Mylodontidae, Megatheriidae y Pampatheriidae (Fig. 2). Tal inmigración sureña refuerza la idea de que los xenarthras fueron fuertes nadadores (De Muizon et al., 2004) y saltadores de islas que superaron los pasos marinos del archipiélago del sur del istmo de Panamá. Al parecer este archipiélago fue un efectivo filtro para la mayoría de los mamíferos norteamericanos a excepción de los Procyonidae, retardando su ingreso a Sudamérica.

La única posible explicación para la migración adelantada por parte de los xenarthras sería la adaptabilidad a las condiciones de vida presentes en el archipiélago que conformaba el Arco de Islas Costa Rica-Panamá; constituidos básicamente por sabanas arboladas y vegetación mixta en una zona basal relativamente cálida y húmeda (Valerio, 2010; Laurito \& Valerio, 2010). Una zona de vida muy similar a la que se supone existió en el centro de Colombia durante el Mioceno Medio en la localidad de La Venta del Grupo Honda (Fig. 1), 
con predominio de tierras húmedas bajas y tropicales (Kay \& Madden, 1997) y donde se registra también el género Scirrotherium. En el caso de los Procyonidae, se asume que su ventaja fue el ser organismos oportunistas altamente adaptables a diferentes ambientes, no limitados por las condiciones climáticas, ni de vegetación.

Fuera de América Central es importante mencionar el hallazgo de otros xenarthras Pre-GABI en América del Norte, como lo son el registro de Megalonyx en el Mioceno de Estados Unidos en las localidades de Draw Fauna, Rattelsnake Formation, Oregon, Hemphilliano temprano (7,05-7,3 Ma sensu Streck \& Grunder, 1995; Protero et al., 2006) y en el Hemphilliano tardío de Optima, Oklahoma (Hirschfeld \& Webb, 1968); Coffe Ranch Local Fauna en Texas con edades de entre 5,0-6,0 Ma (sensu Tedford et al., 1987); o el registro de la especie Megalonyx mathisi en California, hallazgo correlacionado con las capas de Pinole Tuff de edad 5,2 Ma (Hh 3; Tedford et al. [1987, 2004]) y contemporáneas con el registro de Megalonyx de la fauna de Palmetto en Florida (Morgan, 2005). Además, del registro de Megalonyx en las localidades de La Plegaria en el límite entre los estados de Hidalgo y México con una edad Hemphilliano tardío, mayor a los 4,8 Ma (Carranza-Castañeda \& Miller, 2004) y Rancho El Ocote en San Miguel Allende, Guanajuato (Montellano \& Carranza-Castañeda, 1986).

Aunque el género Megalonyx en Norte América había sido comúnmente considerado como un inmigrante temprano de América del Sur (Hirschfeld, 1981), ahora se acepta que es endémico de ese subcontinente (Morgan, 2005) y que evolucionó a partir de Pliometanastes (McDonald \& Naples, 2007).

Por otro lado, en América del Sur hicieron su aparición dos grupos holárticos de Heraldos, la familia Mustelidae y los roedores Sigmodontinae, en el Huayqueriano tardío (alrededor de 5,8 Ma) de la Formación Cerro Azul, provincia de La Pampa en Argentina (Verzi \& Montalvo, 2008; Verzi et al., 2008). Antes de estos hallazgos, el registro más antiguo de un
Mustelidae se dio en el Plioceno tardío de la Formación Vorohué (Marplatense) de acuerdo a Reig (1957) y el de un Cricetidae en el Plioceno temprano de la Formación Monte Hermoso, ambos en Argentina Central (Cuadro 1) (Reig, 1978; Pardiñas et al., 2002).

\section{Intercambio faunístico e Intermitencia} temprana del Istmo de Panamá 4,8-4,5 a 3,5-3,0 Ma

Todos los anteriores registros permiten confirmar que durante el Clarendoniano tardío $(\mathrm{Cl} 2=$ 9.0 Ma aprox.) y el Hemphilliano temprano (Hh1-Hh2 = 8,5 a 6,5 Ma aprox.), arribaron los primeros Heraldos de América del Sur a Norte América y viceversa vía América Central. La evidencia sugiere que ese proceso se dio entre los 9,0 a 5,8 Ma y luego de alguna manera la conexión terrestre entre América del Sur y del Norte, que debe entenderse se dio a lo largo de un antiguo archipiélago, se interrumpió durante casi todo el Hemphilliano tardío (Hh3).

El límite Hemphilliano tardío - Blancano temprano (B11 alrededor de 4,8/4,7 Ma), marca el inicio del cierre o el cierre parcial del istmo de Panamá por el levantamiento continuo del Arco de Islas Costa Rica-Panamá. La circulación de las aguas profundas se mantuvo hasta el Mioceno Superior (Jackson \& Budd, 1996), pero el incremento en la actividad tectónica al final del Mioceno aceleró el proceso de cierre, permitiendo solamente una conexión somera del Pacifico Este con el Caribe, conexiones que permanecieron abiertas hasta alrededor de los 3,5 Ma (Keigwin, 1982; Duque-Caro, 1990a, b).

Ello parece reflejarse en el registro fósil de las localidades de Rancho El Ocote en San Miguel Allende, Guanajuato en el centro de México, donde se citan los géneros Glossotherium (Montellano \& CarranzaCastañeda, 1986), con edades radiométricas cercanas al límite Hemphilliano tardío - Blancano temprano (Hh3-B11 o 4,8/4,7 Ma sensu Flynn 

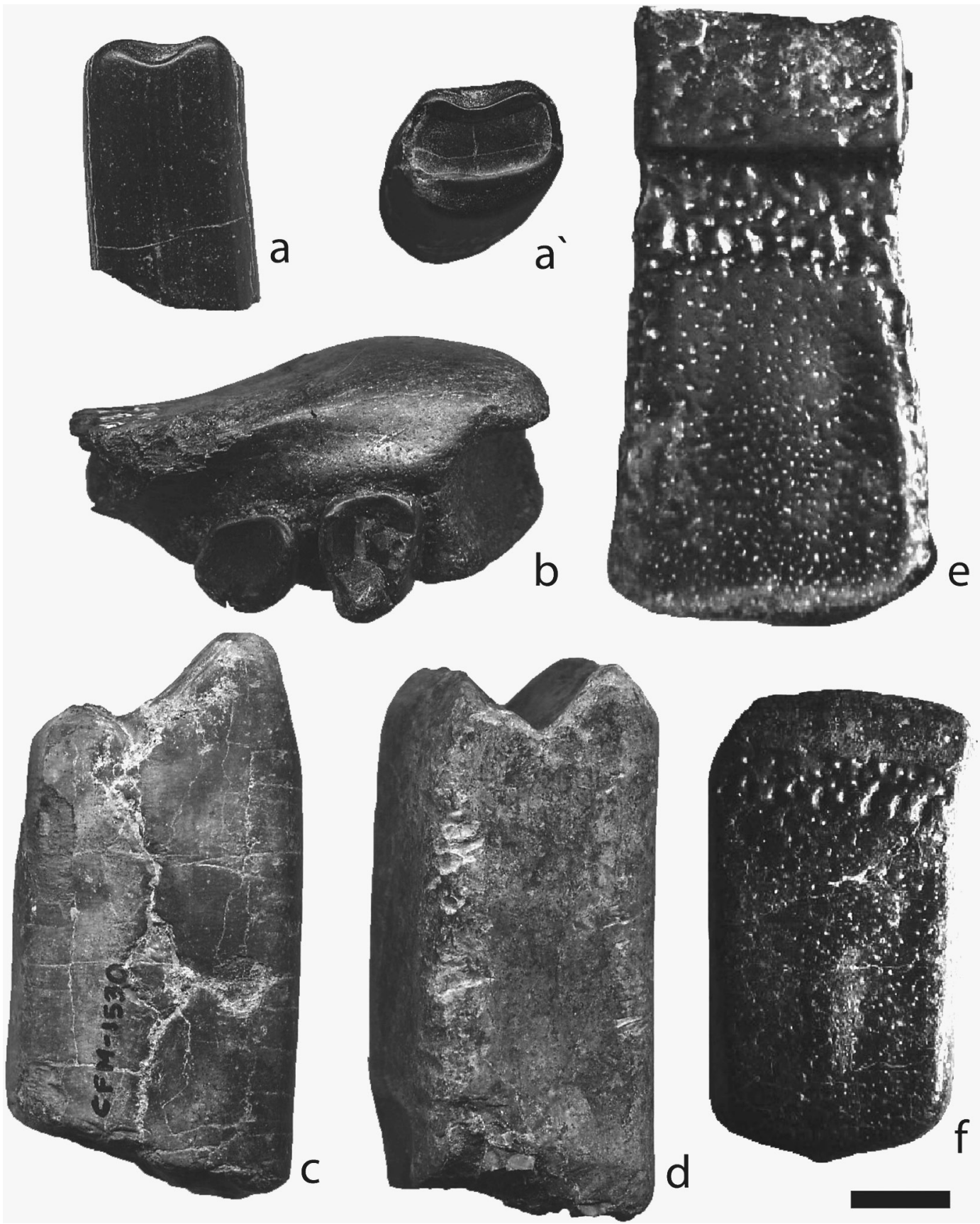

Fig. 2: Xenarthras de la localidad de San Gerardo de Limoncito, Fm. Curré, sur de Costa Rica. a. Megalonychidae correspondiente a Pliometanastes cf. protistus (a, vista mesial, a', vista oclusal); b. Mylodontidae indeterminado; c-d. Megatheriidae indeterminado y Scirrotherium sp., e-f. placas móviles (escala visual $1 \mathrm{~cm}$ ). 
et al., 2005); más tardíamente el Pampatheriidae Plaina (¿?) con edad ligeramente superior a los 3,9 Ma (B11), el Glyptodontidae Glyptotherium ¿? y el roedor caviomorfo Neochoerus (alrededor de 3,6 Ma), todos organismos de abolengo suramericano (a excepción del registro Glyptotherium $¿$ ? , un género norteamericano que evolucionó a partir de un ancestro suramericano y que luego migró de manera inversa hacia América del Sur hace 2,7 Ma, sensu Carlini et al., 2008) (Cuadro 1). Hallazgos todos caracterizados por un registro muy fragmentario de restos, mayoritariamente escudos dérmicos o dientes aislados (CarranzaCastañeda \& Miller, 2004), mismo fenómeno que se observa en la localidad de San Gerardo de Limoncito en la Formación Curré.

El arribo de xenarthras y caviomorfos entre los 4,8 y 3,5 Ma que Carranza-Castañeda \& Miller (2004) comparan con un patrón de inmigración intermitente o una serie de oleadas de arribo y que cabe destacar no parece haberse dado en sentido contrario, es decir de América del Norte hacia América del Sur; este patrón de arribo en oleadas se asocia a un simple acortamiento de los pasos oceánicos por incremento de las superficies de las islas, producto de la conjugación de la elevación del territorio del istmo por el choque del Arco de Islas Costa Rica -Panamá con el noroeste de América del Sur y las caídas del nivel del mar durante el Plioceno, en especial una caída muy fuerte en el Plioceno temprano (Haq et al., 1988) que ha quedado registrada como una discordancia erosiva presente en prácticamente todas las cuencas sedimentarias de Costa Rica durante el Plioceno temprano (Sprechmann et al., 1994).

El componente climático fue crucial para que se diera dicho patrón de intercambio durante el Mioceno tardío y el Plioceno temprano, cuando las asociaciones paleoflorísticas del sur de América Central sugieren ambientes tropicales cálidos y húmedos, bosques lluviosos de zonas bajas sin evidencia de vegetación de altura, árida o seca (Graham \& Dilcher, 1998), condiciones más afines a las existentes en el noroeste de América del Sur (Kay \& Madden, 1997), de allí que facilitara a los saltadores de islas suramericanos arribar al archipiélago de América Central meridional y establecerse. Por el contrario, la vegetación del norte de América Central (Formación Herrería del Plioceno de Guatemala) en esa época, sugiere climas más fríos, vegetación de altura, bosques de pinos y robles con zacates y no está representado el bosque lluvioso de tierras bajas (Grahan \& Dilcher, 1998). A partir de estos datos, se puede deducir que la fauna de Mesoamérica durante el Plioceno Inferior (Blancano temprano) se vio impedida de migrar ante las condiciones climáticas imperantes en el arco de islas Costa Rica-Panamá y el noroeste de América del Sur, condiciones que en definitiva favorecieron a los xenarthras y roedores caviomorfos de América del Sur.

Al parecer el archipiélago del Arco de Islas Costa Rica-Panamá resultó ser un filtro efectivo durante el Plioceno temprano para los migrantes norteamericanos, producto del masivo reajuste ambiental al que eran sometidos y es por ello que su registro en América del Sur no se da hasta el final del Marplatenseano e inicio del Ensenadeano de América del Sur hace unos 2,7 Ma, cuando las condiciones ambientales permitieron el arribo de los primeros tayasuídeos, camélidos, caballos y carnívoros. De hecho la flora del sur de América Central para el Plioceno Medio denota una marcada estacionalidad con una estación seca importante; un desarrollado, mejor definido y extenso bosque tropical seco, producto de la elevación y cierre del portal marino entre Norte y Suramérica (Graham, 1991).

De ahí en adelante, podemos asumir que el cierre efectivo del istmo se da alrededor de los 3,5 a 3,0 Ma en el Blancano medio-tardío y ello es concordante con los datos bioestratigráficos, las sucesiones faunísticas de invertebrados marinos y las secuencias sedimentológicas observadas para el sur de América Central, que permiten establecer que el Istmo de Panamá constituyó un territorio emergido continuo entre $3,5 \mathrm{Ma}$ a $3,1 \mathrm{Ma}$ (Duque-Caro, 1990b; Coates \& Obando, 1996).

El aislamiento de los dos océanos producto del cierre de paso marino centroamericano o estrecho de Bolívar, llevó al sistema climático global hacia una condición cálida, denominada Periodo Plioceno Cálido entre los 4,7- 3,1 Ma (Budyko et al., 1985; Cronin \& Dowsett, 1996) y a un incremento en la salinidad de las aguas superficiales del Caribe entre los 4,7-4,2 Ma según Haug et al. (2001), fundamentados en el análisis comparativo 
de isótopos de oxigeno en foraminíferos plantónicos entre localidades del Pacífico Este (sitio 851 ODP) y el Caribe (sitio 999 ODP), ver también Gussone et al. (2004); condición que se ha mantenido hasta la actualidad. Está situación produjo la posterior extinción de algunos grupos de invertebrados y la migración de otros organismos, como lo son el caso del género Crassostrea en el Caribe (Kirby \& Jackson, 2004) y obligó a la emigración del género Squalus (un grupo de tiburones muy sensibles a los cambios de salinidad y temperatura) fuera del Caribe posterior al Plioceno temprano (Laurito, 1999).

Coates et al. (1992), concluyeron que la divergencia evolutiva entre las faunas marino costeras del Pacífico y el Caribe, acaecieron alrededor de los 3,8 -3,6 Ma, lo que sugiere que el Istmo de Panamá resultó una barrera oceánica efectiva para esa época y que el cierre fue completo de manera adecuada para permitir el amplio intercambio de mamíferos terrestres alrededor de los 2,7 Ma (sensu Webb, 1997).

\section{Algunos casos acerca del GABI en América Central (Cuadro 1)}

En América Central el Gran Intercambio Biótico Americano se caracteriza por una cierta homogeneidad de la fauna, si se le comprara con las faunas Blancano tardío de América del Norte y del Ensenadeano de América del Sur.

En el Blancano tardío de la localidad del río Tomayate, cabe destacar el registro de la familia Ursidae cf. Arctotherium sp. descrito y datado por Soibelzon et al. (2008) y que constituye el registro probablemente más temprano del género en el hemisferio norte de previo al registro Ensenadeano de América del Sur que también es el más antiguo de Argentina (Soibelzon et al., 2005); este registro cambia la suposición previa de que el género Arctotherium era endémico de América del Sur; pero lo cierto es que este género experimentó una cladogénesis importante en el Pleistoceno tardío de América del Sur (Soibelzon et al., 2005).

Para el Irvingtoniano de América Central destacan las localidades de Tomayate (parte superior) en El Salvador (Cisneros, 2005) y la localidad de Bajo de los Barrantes de San Ramón, en Costa Rica, que comparten los registros del Notoungulado suramericano Mixotoxodon larensis van Frank, 1957 (Laurito, 1993; Lucas et al., 1997) descrito inicialmente en Venezuela y poco después en Colombia (van Frank, 1957; De Porta, 1959; Rincón, 2011), muy común en América Central (Leidy, 1886; Gazin, 1957; McGrew, 1942; Stirton \& Gealey, 1949; Webb \& Perrigo, 1984; Espinoza, 1976; Woodburne, 1969) y que se creía restricto a esta área hasta que recientemente Polaco et al. (2004), describen restos de Mixotoxodon sp. en el Pleistoceno de los estados de Michoacán y Veracruz, y más recientemente en el Municipio de Panuco, también en el Estado de Veracruz (Rodríguez-de la Rosa, 2011), todos en México. Por otro lado, Rodrigues do Nascimento (2008) y Rincón (2011), recopilan los registros de Mixotoxodon larensis en los estados de Acre y Rondonia en Brasil y en el departamento de Beni en Bolivia. Recientemente se ha descrito en la provincia de Santiago del Estero en Argentina (Chimento \& Agnolin, 2011) y Rincón (2011) considera a Mixotoxodon larensis una especie intertropical que de acuerdo a los últimos registros se distribuyó entre los $18^{\circ} \mathrm{N}$ y $28^{\circ} \mathrm{S}$ de latitud.

Otro registro Irvingtoniano importante es el Pampatheriidae Holmesina septentrionalis en El Salvador (Aguilar \& Laurito, 2009), se acepta que las especies norteamericanas de Holmesina, evolucionaron de una Holmesina ancestral que en el Plioceno Superior arribó a América del Norte y originó varias especies por cladogénesis (Downing \& White, 1995), para luego reingresar a América del Sur en el Pleistoceno Superior por migración inversa (Scillato-Yané et al., 2005). También es notable en esta época el registro del murciélago Pteronotus cf. parnelli junto con la única evidencia de xenarthras endémicos para América Central conocida hasta ahora, las especies Megalonyx obtusidens Webb \& Perrigo, 1985 y Meizonyx salvadorensis Webb \& Perrigo, 1985 procedentes de la La Barranca del Sisimico.

Un caso interesante lo es el registro del género Pachyarmatherium en el Plioceno tardío y Pleistoceno temprano de la localidad de Buenos 
Aires de Palmares en Costa Rica (Laurito et al., 2005b; Valerio \& Laurito, 2011), previamente descrito en La Florida y Carolina del Norte (Downing \& White, 1995) en esa misma época. Pachyarmatherium se creyó endémico de América del Norte y se acepta que se originó allí a partir de un Glyptatelinae suramericano ancestral (Valerio \& Laurito, 2011), pero al igual que en el caso del género Glyptotherium migra a América del Sur en el Pleistoceno tardío, registrándose en Venezuela y Brasil (Rincón \& White, 2007; Porpino et al., 2008a, b, 2009; Oliveira et al., 2009).

En relación a los proboscídeos de América Central, sobresale el registro más austral del género Mammut en el Pleistoceno de Honduras (Lucas \& Alvarado, 1991); el género Mammuthus con la especie Mammuthus columbi que corresponde al Pacífico Central de Costa Rica (Alvarado, 1986; Lucas et al., 1997) y de edad Rancholabreano (Laurito \& Aguilar, 2006).

Por último, cabe señalar la ausencia del registro fósil del género Stegomastodon que aunque común en el Pleistoceno tardío de Norte América (incluido México) y América del Sur, no se registra en el sur de México, ni en América Central, a pesar que los registros de mastodontes son muy comunes y obvios por el gran tamaño de sus restos; por lo que existe la posibilidad de que el género sea parafilético (cf. Laurito en Arroyo et al., 2007); ésta posición viene a ser reforzada por Mothé et al., (en prensa) y Cozzuol et al. (2012) quienes concluyen que el género "Stegomastodon" de América del Sur está cercanamente relacionado a los géneros Cuvieronius y Rhynchotherium, pero no al género Stegomastodon de Norteamérica.

\section{REFERENCIAS}

AGUILAR, D. \& LAURITO, C., 2009: El armadillo gigante (Mammalia, Xenarthra, Pampatheriidae) del río Tomayate, Blancano tardío-Irvingtoniano temprano, El Salvador, América Central.- Rev. Geol. Amér. Central, 41: 25-35.

ALBERDI, M.T., PRADO, J.L. \& SALAS, R., 2004: The Pleistocene gomphotheres
(Gomphotheriidae, Proboscidea) from Peru.- Neues Jarhbuch für Geologie un Paläontolgie abhandlungen, 231: 423-452.

ALVARADO, G., 1986: Hallazgo de megamamíferos fósiles en Costa Rica.- Rev. Geol. Amér. Central, 4: 1-46.

ARROYO-CABRALES, J., POLACO, O.J., LAURITO, C.A., JOHNSON, E., ALBERDI, M.T. \& VALERIO, A.L., 2007: The Proboscideans (Mammalia) from Mesoamerica.- En: AGENBROAD, L., HAYNES, G., JOHNSON, E. \& PALOMBO, M.R. (eds): World of Elephants 2: Selected papers from the 2nd Congress, Mammoth Site of Hot Springs.Quat. Intern. 169-170: 17-23.

BUDYKO, M.I., RONOV, A.B. \& YANSHIN, A.L., 1985: The history of the Earth atmosphere.- 139 págs. Springer-Verlag, Berlin.

CADENA, E.A., 2009: New Cretaceous and Cenozoic fossil turtles from Colombia and Panama; systematic, paleontology, phylogenetical and paleobiogeographical implications.- 133 págs. Universidad de Florida, Florida [Tesis M.Sc.].

CADENA, E., BOURQUE, J.R., RINCON, A.F., BLOCH, J.I., JARAMILLO, C.A. \& MACFADDEN , B.J., 2012: New Turtles (Chelonia) from the Late Eocene Through Late Miocene of the Panama Canal Basin.J. Paleontol. 86(3): 539-557.

CAMPBELL, K.E., HEIZLER, M., FRAILEY, C.D., ROMERO PITMAN, L., \& PROTHERO, D.R., 2001: Upper Cenozoic chronostratigraphy of the southwestern Amazon Basin.- Geology, 29: 595-598.

CARLINI, A., ZURITA, A. \& AGUILERA, O., 2008: North American Glyptodontines (Xenarthra, Mammalia) in Upper Pleistocene of northern South America.- Paläontologische Zeitschrift, 82(2): 125-138. 
CARRANZA-CASTAÑEDA, O. \& MILLER, W., 2004: Late Tertiary Terrestrial Mammals from Central Mexico and their relationship to South American Inmigrants.- Rev. Brasileira de Paleont. 7(2): 249-261.

CHIMENTO, N.R. \& AGNOLIN, F.L., 2012: Mamíferos del Pleistoceno Superior de Santiago del Estero (Argentina) y sus afinidades paleobiogeográficas.- Papéis Avulsos Zoologia, 51(6): 83-100.

CISNEROS, J.C., 2005: New Pleistocene Vertebrate Fauna from El Salvador.- Rev. Bras. de Paleontol., 8(3): 239-255.

COATES, A.G. \& OBANDO, J.A., 1996: The geologic evolution of the Central American isthmus.- En: JACKSON, J.B.C., BUDD, A.F. \& COATES, A.G. (eds.): Evolution and environment in tropical America.- The Univ. Chicago Press, Chicago: 21-56.

COATES, A.G., COLLINS, L.S., AUBRY, M. \& BERGGREN, W.A., 2004: The Geology of the Darien, Panama, and the late MiocenePliocene collision of the Panama arc with northwestern South America.- Geol. Soc. Amer. Bull. 116: 1327-1344.

COATES, A.G., AUBRY, M., BERGGREN, W.A., COLLINS, L.S., \& KUNK, M., 2003: Early Neogene history of the Central American arc from Bocas del Toro, western Panamá.- Geol. Soc. Amer. Bull. 115: 271-287.

COATES, A.G., JACKSON, J.B.C., COLLINS, L.S., CRONIN, T.M., DOWSETT, H.J., BYBELL, L.M., JUNG, P. \& OBANDO, J.A., 1992: Closure of the Isthmus of Panama: The near-shore marine record of Costa Rica and western Panama.- Geol. Soc. of Amer. Bull. 104: 814-828.

COZZUOL, M.A., MOTHÉ, D. \& AVILLA, L.S.A., 2012: Critical apprasial of the phylogenetic proposals for the South American Gomphotheriidae (Proboscidea: Mammalia).- Quat. Intern. 255: 36-41.

CRONIN, T.M. \& DOWSETT, H.J., 1996: Biotic and oceanographic response to the Pliocene closing of the Central American isthmus. - En: JACKSON, J.B., BUDD, A.F. \& COATES, A.G. (eds.): Evolution and Environment in Tropical America.- The Univ. Chicago Press, Chicago: 76-104.

DE MUIZON, C., MCDONALD, H.G., SALAS, R. \& URBINA, M., 2004: The evolution of feeding adaptations of the aquatic sloth Thalassocnus.- J. Vert. Paleontol. 24: 398-410.

DE PORTA, J., 1959: Nueva subespecie de Toxodóntido del Cuaternario de Colombia.Bol. de Geol. Univ. Industrial de Santander, 3: 55-61.

DOMNING, D.P., EMRY, R.J., PORTELL, R.W., DONOVAN, S.K. \& SCHINDLER, K.S., 1997: Oldest West Indian Land Mammal: Rhinocerotoid Ungulate from the Eocene of Jamaica.- J. Vert. Paleontol. 17(4): 638-641.

DOWNING, K. \& WHITE, R., 1995: The cingulates (Xenarthra) of Leisey Shell Pit local fauna (Irvingtonian), Hillborough County, Florida.- Bull. Florida Mus. Nat. Hist. 37: 375-396.

DUQUE-CARO, H., 1990a: Estratigrafía, paleoceanografía y paleobiogeografía de la cuenca del Atrato y la evolución del Istmo de Panamá.- Inst. Nac. Invest. Geol. Mineras. Bol. Geol. 31(1): 3-46.

DUQUE-CARO, H., 1990b: El Bloque Chocó en el Noroccidente Suramericano: Implicaciones estructurales, tectonoestratigráficas y paleogeográficas.- Inst. Nac. Invest. Geol. Mineras. Bol. Geol. 31(1): 47-71. 
ESPINOSA, J., 1976: Excavaciones arqueológicas en "El Bosque". Informe No.1.- 70 págs. Instituto Geográfico Nacional, Managua, Nicaragua.

FERRETTI, M.P., 2008: A review of South American proboscideans.- En: LUCAS, S.G., MORGAN, G.S., SPIELMANN, J.A. \& PROTHERO, D.R. (eds.): Neogene Mammals.- New Mex. Mus. Nat. Hist. Sci. Bull. 44: 381-391.

FERRUSQUIA-VILLAFRANCA， I., 2003: Mexico's Middle Miocene mammalian assemblages: An overview. - En: FLYNN, L. (ed.): Vertebrate Fossils and Their Context: Contributions in Honor of Richard H. Tedford. - Bull. Amer. Mus. Nat. Hist. 279: 321-347.

FLYNN, J., KOWALLIS, B., NUÑEZ, C., CARRANZA-CASTAÑEDA, O., MILLER, W., SWISHER III, C. \& LINDSAY, E., 2005: Geochronology of Hemphillian-Blancan aged strata, Guanajuato, Mexico, and implications for timing of the Great American Biotic Interchange.- J. of Geol.113: 287-307.

GAZIN, C.L., 1957: Exploration for the remains of giant ground sloths in Panama.Smithsonian Institution, Annual Report, 1956: 314-354.

GRAHAM, A., 1991: Studies in Neotropical Paleobotany. X. The Pliocene communities of Panama: composition, numerical representations, and paleocommunity paleoenvironmental reconstructions.An. of the Missouri Botanical Garden, 78: $465-475$.

GRAHAM, A. \& DILCHER, D.L., 1998: Studies in Neotropical Paleobotany. XII. A Palynoflora from the Pliocene Rio Banano Formation of Costa Rica and the Neogene Vegetation of Mesoamerica.- Amer. J. of Botany, 85(10): 1426-1438.
GUSSONE,N.,EISENHAUER,A,TIEDEMANN, R., HAUG, G.H., HEUSER, A., BOCK, B., NÄGLER, T. \& MÜLLER, A., 2004: Reconstruction of Caribbean Sea surface temperature and salinity fluctuations in response to the Pliocene closure of the Central American Gateway and radiative forcing, using $\delta^{4 / 40} \mathrm{Ca}, \delta^{18} \mathrm{O}$ and $\mathrm{Mg} / \mathrm{Ca}$ ratios.- Earth and Planetary Sci. Letters, 227(2004): 201-214.

HAQ, B.U., HARDENBOL, J. \& VAIL, P.R., 1988: Mesozoic and Cenozoic chronostratigraphy and cycles of sea-level change.- En: WILGUS, C.K., HASTINGS, B., KENDALL, C.G.St.C, POSAMENTIER, H.W., ROSS, C.A. \& VAN WAGONER, J.C. (eds.): Sea Level Changes: An Integrated Approach. SEPM Spec. Publ. 42: 71-108.

HAUG, G.H., TIEDEMANN, R., ZAHN, R. \& RAVELO, A.C., 2001: Role of Panama uplift on oceanic freshwater balance.Geology, 29: 207-210.

HEAD, J.J., RAZA, S.M. \& GINGERICH, P.D., 1999: Drazinderetes tethyensis: a new large trionychid (Reptilia:Testudines) from the marine Eocene Drazinda Formation of the Sulaiman Range, Punjab (Pakistan).Contrib. from the Mus. of Paleontol. Univ. of Michigan, 30: 199-214.

HEAD, J.J., AGUILERA, O. \& SÁNCHEZVILLAGRA, M.R., 2006: Past colonization of South America by trionychid turtles: Fossil evidence from the Neogene of Margarita Island, Venezuela.- J. of Herpetology, 40(3): 380-383.

HELGEN, K.M. \& WILSON, D.E., 2003: Taxonomic status and conservation relevance of the raccoons (Procyon spp.) of the West Indies.- J. of Zoology, 259(1): 69-76.

HIRSCHFELD, S.E., 1981: Pliometanastes protistus (Edentata, Megalonychidae) from 
Knight's Ferry, California, with discussions of early Hemphillian megalonychidos.- PaleoBios, 36: 1-16.

HIRSCHFELD, S.E., 1985: Ground sloths from the Friasian La Venta Fauna, with additions to the pre-Friasian Coyaima Fauna of Colombia, South America.Univ. of California Publications, Geol. Sci. 128: 1-91.

HIRSCHFELD, S.E. \& WEBB, S.D., 1968: PIioPleistocene megalonychid sloths of North America. - Bull. of the Fla. State Mus. Biol. Sci. 12(5): 213-296.

HORNE, G.S., 1994: A Mid Cretaceous ornithopode from Central Honduras.- J. Vert. Paleontol. 14(1): 147-150.

ITURRALDE-VINNENT, M.A. \& MACPHEE, R.D.E., 1999: Paleogeography of the Caribbean Region: Implications for Cenozoic Biogeography. - Bull. of the Amer. Mus. of Natural History, 238: 1-95.

JACKSON, J.B. \& BUDD, A.F., 1996: Evolution and Environment: Introduction and Overview. - En: JACKSON, J.B., BUDD, A.F. \& COATES, A.G. (eds.): Evolution and Environment in Tropical America.The Univ. of Chicago Press, 1-20.

JOHNSON, D.L., 1980: Problems in the Land Vertebrate Zoogeography of Certain Islands and the Swimming Powers of Elephants.J. Biogeography, 7(4): 383-398.

KAY, R.F. \& MADDEN, R.H., 1997: Mammals and rainfall: paleoecology of the middle Miocene at La Venta (Colombia, South America).- J. Human Evol. 32: 161-199.

KEIGWIN, L.D., Jr., 1982: Isotopic paleoceanography of the Caribbean and East Pacific: role of Panama uplift in late Neogene time.- Science, 217: 350-353.
KELLOGG, J.N. \& VEGA, V., 1995: Tectonic development of Panama, Costa Rica and the Colombian Andes: Constraints from Global Positioning System geodetic studies and gravity.- En: MANN, P. (ed.): Geologic and Tectonic Development of the Caribbean Plate Boundary in Southern Central America.Geol. Soc. Amer. Spec. paper 295: 75-88.

KIRBY, M.X. \& JACKSON, J.B.C., 2004: Extinction of a fast-growing oyster and changing ocean circulation in Pliocene tropical America.- Geology, 32: 1025-1028.

KIRBY, M.X. \& MACFADDEN, B.J., 2005: Was southern Central America an archipelago or a peninsula in the middle Miocene? A test using land-mammal body size.Palaeogeography, Palaeoclimatology, Palaeoecology, 228: 193-202.

LAURITO, C., 1993: Análisis topológico y sistemático del Toxodonte de Bajo de los Barrantes, provincia de Alajuela, Costa Rica.- Rev. Geol. Amér. Central, 16: 61-68.

LAURITO, C., 1999: Los seláceos fósiles de la localidad de Alto Guayacán (y otros ictiolitos asociados), Mioceno Superior-Plioceno Inferior de la Formación Uscari, provincia de Limón, Costa Rica.- 168 págs. Laurito, C. (ed.), San José.

LAURITO, C. \& AGUILAR, D., 2006: El registro de Mammuthus (Proboscidea, Elephantidae) en la República de El Salvador, América Central.- Rev. Geol. Amér. Central, 34-35: 73-81.

LAURITO, C. \& VALERIO, A., 2010: Los caballos fósiles de la Formación Curré, cantón de Coto Brus, Costa Rica.- 131 págs. Mus. Nac.Costa Rica, San José.

LAURITO, C., VALERIO, A. \& PÉREZ, E., 2005b: Los xenarthras fósiles de la localidad de Buenos Aires de Palmares (Blancano tardío-Irvingtoniano temprano), 
provincia de Alajuela, Costa Rica.- Rev. Geol. Amér. Central, 33: 83-90.

LAURITO, C., VALERIO, A., GÓMEZ, L., MEAD, J., PÉREZ, E. \& PÉREZ, L., 2005a: A Trionychidae (Reptilia: Testudines, Cryptodira) from the Pliocene of Costa Rica, Southern Central America.Rev. Geol. Amér. Central, 32: 7-11.

LEIDY, J., 1886: Toxodon and other remains from Nicaragua, C.A.- Proc. of the Academy of Natural Sciences, Philadelphia, 38: 275277.

LUCAS, S.G. \& ALVARADO, G.E., 1991: El hallazgo más austral de Mammut americanum: el caso del mastodonte de San Pedro Sula, Honduras.- Rev. Geol. Amér. Central, 13: 85-89.

LUCAS, S., ALVARADO, G. \& VEGA, E., 1997: The Pleistocene mammals of Costa Rica. J. Vert. Paleontol. 17(2): 413-427.

MACFADDEN, B.J., 2006: Early Pliocene (latest Hemphillian) horses from the Yepómera Local Fauna,Chihuahua,Mexico.-En:CARRANZACASTAÑEDA, O. \& LINDSAY, E.H. (eds.): Advances in late Tertiary vertebrate paleontology in Mexico and the Great American Biotic Interchange.- Univ. Nac. Autónoma de México, Inst. de Geol. y Centro de Geociencias, Pub. Espec. 4: 33-43.

MACFADDEN, B.J., KIRBY, M.X., RINCÓN,A., MONTES, C., MORON, S., STRONG, N. \& JARAMILLO, C.A., 2010: Extinct peccary "Cynorca" occidentale (Tayassuidae, Tayassuinae) from the Miocene of Panama and correlations to North America. - J. Paleontol. 84(2): 288-298.

MACPHEE, R.D.E., 2005: 'First' appearances in the Cenozoic land-mammal record of the Greater Antilles: significance and comparison with South American and Antarctic records.- J. Biogeography, 32: 551-564.
MACPHEE, R.D.E. \& ITURRALDEVINENT, M.A., 1994: First Tertiary land mammal from Greater Antilles: an Early Miocene sloth (Xenarthra, Megalonychidae) from Cuba. - Amer. Mus. Novitates, 3094: 1-13.

MACPHEE, R.D.E. \& ITURRALDE-VINENT, M.A., 1995: Origin of the Greater Antillean land mammal fauna, 1: new Tertiary fossils from Cuba and Puerto Rico.- Amer. Mus. Novitates, 3141: 1-31.

MACPHEE, R.D.E., ITURRALDE-VINENT, M.A. \& GAFFNEY, E.S., 2003: Domo de Zaza, an Early Miocene vertebrate locality in south-central Cuba, with notes on the tectonic evolution of Puerto Rico and the Mona Passage.- Amer. Mus. Novitates, 3394: 1-42.

MCDONALD, H.G., 2005: Paleoecology of the extinct Xenarthras and The Great American Biotic Interchange.- En: HULBERT, R.C., MORGAN, G.S. \& BASKING, J.A. (eds): Cenozoic Vertebrates of the Americas. Paper to Honor David S. Webb.- Bull. Fla. Mus. Nat. Hist. 45(4): 319-340.

MCDONALD, H.G. \& NAPLES, V.L., 2007: Chapter 10: Xenarthra.- En: JANIS, C.M., GUNNELL, G.F. \& UHEN, M.D. (eds): Evolution of Tertiary Mammals of North America, Vol. 2. Cambridge Univ. Press, 147-160.

MCGREW, P.O., 1942: Special correspondence: Field Museum Paleontological expedition to Honduras.- Science, 96:85.

MONTELLANO, M. \& CARRANZACASTAÑEDA, O., 1986: Descripción de un milodóntido del Blancano temprano de la Mesa Central de México.- Rev. Univ. Nac. Autónoma de México, 6: 193-203.

MORGAN, G.S., 2005: The Great American Biotic Interchange in Florida.- Bull. Florida Mus. Nat. Hist. 45(4): 271-311. 
MOTHÉ, D., AVILLA, L.S.A., COZZUOL, M.A. \& WINCK, G.R., en prensa: Taxonomic revision of the Quaternary gomphotheres (Mammalia: Proboscidea: Gomphotheriidae) from the South American lowlands. - Quat. Intern. 2011: 1-6.

OLIVEIRA, É.V., FRANCA-BARRETO, A.M. \& DA SILVA ALVES, R., 2009: Aspectos sistemáticos, paleobiogeográficos e paleoclimáticos dos mamíferos quaternários de Fazenda Nova Gaea.- J. Geoscience, 5(2): 75-85.

OLSON, E.C. \& MCGREW, P.O., 1941: Mammalian fauna from the Pliocene of Honduras.- Bull. Geol. Soc. Amer. 52: 1219-1244.

PARDIÑAS, U.F.J., D'ELÍA, G. \& ORTIZ, P.E., 2002: Sigmodontinos fósiles (Rodentia, Muroidea, Sigmodontinae) de América del Sur; estado actual de su conocimiento y prospectiva.- Mastozoología Neotropical, 9: 209-252.

PASCUAL, R., 2006: Evolution and geography: The Biogeographic History of South American Land Mammals.- Ann. Missouri Bot. Gard. 93: 209-230.

PATTERSON, B. \& PASCUAL, R., 1968: Evolution of mammals on southern continents. The fossil mammal fauna of South America.- The Quarterly Rev. of Biol. 43: 409-451.

PATTERSON, B. \& PASCUAL, R., 1972: The fossil mammal fauna of South America. En: KEAST, A., ERK, F.C. \& GLASS, B. (eds): Evolution, Mammals, and Southern Continents.- State Univ. New York Press, Albany, 247-309.

PINDELL, J., 1994: Evolution of the Gulf of Mexico and the Caribbean.- En: DONOVAN, S.K. \& JACKSON, T.A. (eds): Caribbean geology, an intro- duction.- The Univ. of the West Indies Publishers Assoc.: 13-40.

POLACO, O., GUZMÁN, A. \& TAPIA, G., 2004: Occurrence of Toxodonts in the Pleistocene of México.- Current Res. in the Pleistocene, 21:113-115.

PORPINO, K. DE O., FERNICOLA, J.C. \& BERQVIST, L.P., 2008a: Pachyarmatherium (Xenarthra, Cingulata) remains from the Late Pleistocene of Northeastern Brazil and its phylogenetic affinities. VI Simpósio Brasileiro de Paleontología de Vertebrados.- Bol. de Resumos, Ribeirão Preto, USP, 163-164.

PORPINO, K. DE O., FERNICOLA, J.C. \& BERQVIST, L.P., 2008b: A new species of Pachyarmatherium (Xenarthra: Cingulata) from the late Pleistocene of northeastern Brazil and its phylogenetic affinities.- J. Vert. Paleontol. 28(3) Abstract 128A.

PORPINO, K. DE O., FERNICOLA, J.C. \& BERQVIST, L.P., 2009: A new Cingulate (Mammalia: Xenarthra), Pachyarmatherium brasiliense $\mathrm{sp}$. nov. from the Late Pleistocene of Northeastern Brazil.- J. Vert. Paleontol. 29(3): 881-893.

PRITCHARD, P., 1984: Evolution and zoogeography of South American turtles.- Studia Geologica Salmanticensia, 1: 225-233.

PROTHERO, D.R., HOFFMAN, J.M. \& FOSS, S.E., 2006: Magnetic stratigraphy of the upper Miocene (Hemphillian) Rattlesnake Formation, central Oregon.- PaleoBios, 26: $37-42$.

REIG, O.A., 1957: Un mustélido del género Galictis del Eocuartario de la Provincia de Buenos Aires. - Ameghiniana, 1: 33-47.

REIG, O.A., 1978: Roedores cricétidos del Plioceno Superior de la provincia de Buenos Aires (Argentina).- Publicaciones 
del Mus. Municipal de Cien. Naturales «Lorenzo Scaglia», 2(8): 164-190.

REIG, O.A., 1981: Teoría y desarrollo de la fauna de mamíferos de América del Sur.162 págs. Monographiae Naturae. Mus. Municipal de Cien. Naturales «Lorenzo Scaglia», Mar del Plata, Argentina.

RINCÓN, A.D., 2011: New remains of Mixotoxodon larensis Van Frank 1957 (Mammalia: Notoungulata) from Mene de Inciarte tar pit, North-Western Venezuela.Interciencia, 36(12): 894-899.

RINCÓN, A.D. \& WHITE, R., 2007: Los Xenarthra Cingulata del Pleistoceno tardío (Lujanense) de Cerro Misión, Estado de Falcón, Venezuela.- Bol. Soc. Venezolana Espeleol. 41: 2-12.

RINCON, A.F., BLOCH, J., MACFADDEN, B., SUAREZ, S. \& JARAMILLO, C., 2012: New Floridatragulines (Mammalia, Camelidae) from the Early Miocene Las Cascadas Formation, Panama.- J. Vert. Paleont. 32(2):456-475

ROBINSON, E., 1988: Early Tertiary larger foraminifera and platform carbonates of the northern Caribbean.- En: BARKER, L.H. (ed.): Transactions of the 11th Caribbean Geological Conference.Energy \& Natural Resources Division, Barbados: 501-512.

RODRIGUES DO NASCIMENTO, E., 2008: Os Xenarthra Pilosa (Megatheriidae), Notoungulata (Toxodontidae) e Proboscidea (Gomphotheriidae) da formação Rio Madeira, Pleistoceno Superior, Estado de Rondônia, Brasil.- 113 págs. Universidade Federal do Rio Grande do Sul. Porto Alegre [Tesis M.Sc.].

RODRÍGUEZ-DE LA ROSA, R.A., GUZMÁNGUTIÉRREZ, J.R. \& ORTEGAHURTADO DE MENDOZA, C., 2011:
A new occurrence of toxodonts in the Pleistocene of México.- Current Res. in the Pleistocene, 28: 29-30.

SÁNCHEZ-VILLAGRA, M.R. \& SCHEYER, T.M., 2010: Fossil turtles from the northern Neotropics: the Urumaco sequence fauna and finds from other localities in Venezuela and Colombia.En: SANCHEZ-VILLAGRA, M.R., AGUILERA, O.A. \& CARLINI, A.A. (eds): Urumaco and Venezuelan Palaeontology. The Fossil Record of the Northern Neotropics.- Indiana University Press, Bloomington: 173-191.

SÁNCHEZ-VILLAGRA, M.R., ASHER, R.J., RINCÓN, A.D., CARLINI, A., MEYLAN, P. \& PURDY, W., 2004: New faunal reports for the cerro $\mathrm{La}$ Cruz locality (Lower Miocene), NorthWestern Venezuela.- En: SÁNCHEZVILLAGRA, M.R. \& CLACK, J.A. (eds): Fossil of the Miocene Castillo Formation, Venezuela.- Contributions on Neotropical Paleontol. Spec. Papers in Paleontol. 71: 105-112.

SCILLATO-YANÉ, G.J., CARLINI, A.A., TONNI, E.P. \& NORIEGA, J.I., 2005: Paleobiogeography of the late Pleistocene pampatheres of South America.- J. South American Earth Sciences, 20: 131-138.

SIMPSON, G.G., 1956: Zoogeography of West Indian Land Mammals.- Amer. Mus. Novitates, 1759: 1-28.

SLAUGHTER, R.H., 1981: Anew genus of geomyid rodent from the Miocene of Texas and Panama. - J. Vert. Paleontol. 1: 111-115.

SOIBELZON, L.H., TONNI, E.P. \& BOND, M., 2005: The fossil record of the South American Short-faced bears (Ursidae, Tremarctinae).- J. South Amer. Earth Sciences, 20: 105-113. 
SOIBELZON, L.H., ROMERO, M., AGUILAR, D. \& TARTARINI, V., 2008: A Blancan (Pliocene) short-faced bear fromElSalvador and its implications for Tremarctines in South America.- N. Jb. Geol. Paläont. Abh 250(1): 1-8.

SPRECHMANN, P., ASTORGA, A., CALVO, C. \& FERNÁNDEZ, J.A., 1994: Stratigraphic chart of the sedimentary basins of Costa Rica, Central America.- Profil 7: 427-433.

STIRTON, R.A. \& GEALEY, W.K. 1949: Reconnaissance geology and vertebrate paleontology of El Salvador, Central America.- Geol. Soc. Amer. Bull. 60: $1731-1764$.

STRECK, M.J. \& GRUNDER, A.L., 1995: Crystallization and welding variations in a widespread ignimbrite sheet: the Rattlesnake Tuff, eastern Oregon, U.S.A.Bull. Volcanol. 57: 151-169.

SYKES, L.R., MCCANN, W.R. \& KAFKA, A.L., 1982: Motion of the Caribbean Plate during the last 7 million years and implications for earlier Cenozoic movements.- J. Geophys. Res. 87(10): 656-676.

TEDFORD, R.H., GALUSHA, T., SKINNER, M.F., TAYLOR, B.E., FIELDS, R.W., MACDONALD, J.R., RENSBERGER, J.M., WEBB, S.D. \& WHISTLER, D.P., 1987: Faunal succession and biochronology of the Arikareean through Hemphillian interval (Late Oligocene through earliest Pliocene epochs) in North America.- En: WOODBURNE, M.O. (ed.): Cenozoic Mammals of NorthAmerica: Geochronology and Biostratigraphy.- University of California Press, Berkeley, 153-210.

TEDFORD, R.H., ALBRIGHT III, L.B., BARNOSKY, A.D., FERRUSQUIAVILLAFRANCA, I., HUNT JR., R.M., STORER, J.E., SWISHER III, C.C., VOORHIES, M.R., WEBB, S.D. \&
WHISTLER, D.P., 2004: Mammalian biochronology of the Arikareean through Hemphillian interval (Late Oligocene through early Pliocene epochs).- En: WOODBURNE, M.O. (ed.): Late Cretaceous and Cenozoic Mammals of North America.- Columbia Univ. Press, New York, 169-231.

VALERIO, A.L., 2010: Paleontología, bioestratigrafía y paleoecología de los caballos fósiles de la Formación Curré en el cantón de Coto Brus, Costa Rica (análisis basado en material dental).- 353 págs. Univ. de Costa Rica, San José [Tesis Lic.].

VALERIO, A.L. \& LAURITO, C.A., 2011: Nuevos hallazgos de Mammalia, Xenarthra (Cingulata) y confirmación del registro de Pachyarmatherium leiseyi Downing \& White, 1995 en la localidad de Buenos Aires de Palmares, provincia de Alajuela, Costa Rica.Rev. Geol. Amér. Central, 44: 131-139.

VALERIO, A.L. \& LAURITO, C.A., 2012: Cetáceos fósiles (Mammalia, Odontoceti, Eurhinodelphinoidea, Inioidea, Physeterioidea) de la Formación Curré, Mioceno Superior (Hemphilliano temprano tardío) de Costa Rica.- Rev. Geol. Amér. Central, 46: 151-160.

VAN FRANK, R., 1957: A fossil collection from northern Venezuela. 1. Toxodontidae (Mammalia, Notoungulata).- Amer. Mus. Novitates, 1850: 1-38.

VÉLEZ-JUARBE, J., BROCHU, C.A. \& SANTOS, H., 2007: A gharial from the Oligocene of Puerto Rico: transoceanic dispersal in the history of a non-marine reptile.- Proc. of The Royal Soc. Biol Sci. 274(1615): 1245-1254.

VERZI，D.H. \& MONTALVO, C.I., 2008: The oldest South American Cricetidae (Rodentia) and Mustelidae (Carnivora): Late Miocene faunal turnover in cen- 
tral Argentina and the Great American Biotic Interchange.- Palaeogeography, Palaeoclimatology, Palaeoecology, 267: 284-291.

VERZI,D.H.,MONTALVO,C.I.\&DESCHAMPS, C.M., 2008: Biostratigraphy and biochronology of the Late Miocene of central Argentina: evidence from rodents and taphonomy.- Geobios, 41: 145-155.

WEBB, S.D., 1976: Mammalian faunal dynamics of the Great American Interchange.Paleobiology, 2: 216-234.

WEBB, S.D., 1985: Late Cenozoic mammal dispersals between the Americas.- En: STEHLI, F.G. \& WEBB, S.D. (eds): The Great American Biotic Interchange.Topics in Geobiology, 4: 357-386.

WEBB, S.D., 1989: Osteology and relationships of Thinobadistes segnis, the first mylodont sloth in North America. - En: REDFORD, K.H. \& EISENBERG, J.F. (eds.): Advances in Neotropical Mammalogy.- The Sandhill Crane Press, 469-532.

WEBB, S.D., 1997: The great American faunal inter-change.- En: COATES, A.G. (ed.): Central America: A natural and cultural history.- Yale Univ. Press, New Haven, Connecticut, 97-122.
WEBB, S.D., 2006: The Great American Biotic Interchange, Patterns and Processes.- Ann. Missouri Bot. Gard. 93: 245-257

WEBB, S.D. \& PERRIGO, S.C., 1984: Late Cenozoic vertebrates from Honduras and $\mathrm{El}$ Salvador.- J. Vert. Paleontol. 4(2): 237-254.

WEBB, S.D. \& PERRIGO, S.C., 1985: New Megalonychid sloths from El Salvador.En: MONTGOMERY, G.G. (ed.): The Evolution and Ecology of Armadillos, Sloths, and Vermilinguas.- Smithsonian Institution Press: Washington, 13-120.

WHITE, J., \& MACPHEE, R.D.E., 2001: The sloths of the West Indies: a systematic and phylogenetic review.- En: WOODS, C.A. $\&$ SERGILE, F.E. (eds): Biogeography of the West Indies: Patterns and Perspectives [2 $2^{\mathrm{a}}$ ed.]. Boca Raton, FL, 201-236.

WHITEMORE, F.C. \& STEWART, R.H.; 1965: Miocene mammals and Central American seaways.- Science, 148: 180-185.

WOOD, R.C. \& PATTERSON, B., 1973: A fossil Trionychid turtle from South America.Brevoria, 405: 1-10.

WOODBURNE, M.O., 1969: A Late Pleistocene occurrence of the Collared Peccary, Dicotyles tajacu, in Guatemala.- J. Mammalogy, 50(1): 121-125. 


\section{APÉNDICE}

Fauna local de San Gerardo de Limoncito, Formación Curré, Costa Rica

Clase CHONDRICTHYES [tiburones y rayas]

Subclase ELASMOBRANCHII

Orden LAMNIFORMES

Familia LAMNIDAE

Isurus desori (Agassiz, 1843) (Sismonda, 1849)

Orden CARCHARHINIFORMES

Familia HEMIGALEIDAE

Hemipristis serra Agassiz, 1843

Familia CARCHARHINIDAE

Carcharhinus priscus (Agassiz, 1843)

Carcharhinus longimanus (Poey, 1861)

Isogomphodon acuarius (Probst, 1879)

Isogomphodon caunellensis (Cappetta, 1970)

Familia SPHYRNIDAE

Sphyrna arambourgi Cappetta, 1970

Orden MYLIOBATIFORMES

Familia DASYATIDAE

Dasyatis cavernosa (Probst, 1877)

Familia RHINOPTERIDAE

Rhinoptera sp.

Myliobatis sp.

Aetobatus arcuatus Agassiz (1843)

Clase OSTEICHTHYES [peces óseos]

Subclase ACTINOPTERYGII

Orden SILURIFORMES

Familia ARIIDAE

Arius sp.

Orden PERCIFORMES

Familia SPARIDAE

Sparus sp.

Clase SARCOPTERYGII [peces de aletas lobuladas y tetrápodos]

Subclase TETRAPODA

Orden TESTUDINES (=CHELONIA)
Familia TRIONYCHIDAE

Apalone sp.

Familia EMYDIDAE

Subfamilia EMYDINAE

Género y especies indet.

Subfamilia DEIROCHELYINAE

Género y especies indet.

Familia TESTUDINIDAE

Subfamilia XEROBATINAE

Género y especies indet.

Orden CROCODYLIA

Familia CROCODYLIDAE

Crocodylus sp.

Gavialosuchus americanus (Sellards, 1915)

Orden CINGULATA

Familia PAMPATHERIIDAE

Scirrotherium sp.

Orden PILOSA

Familia MYLODONTIDAE

Género y especie indet.

Familia MEGATHERIIDAE

Género y especie indet.

Familia MEGALONYCHIDAE

Pliometanastes cf. P. protistus Hirschfeld

\& Webb 1968

Orden ARTIODACTYLA

Familia TAYASSUIDAE

Género y especie indet.

Familia CAMELIDAE

Hemiauchenia sp. 1

Hemiauchenia sp. 2

Orden CETACEA

Familia EURHINODELPHINIDAE

Eurhinodelphis sp.

Familia INIIDAE

Goniodelphis sp.

Familia PHYSETERIDAE

Orycterocetus sp. 
Familia KENTRIODONTIDAE

Hadrodelphis sp.

Orden PERISSODACTYLA

Familia EQUIDAE

Protohippus gidleyi Hulbert, 1988

Calippus (Grammohippus) hondurensis
(Olson \& McGrew, 1941)

Dinohippus mexicanus (Lance, 1950)

Orden URANOTHERIA

Familia GOMPHOTHERIIDAE

Gomphotherium hondurensis (Frick

1933) Lucas \& Morgan, 2008 